S sciendo

\title{
What Is Structuralism?
}

\author{
M. S. Lourenço \\ LanCog, Universidade de Lisboa
}

Disputatio Vol. 3, No. 27

November 2009

DOI: $10.2478 /$ disp-2009-0012

ISSN: 0873-626X 


\title{
What is structuralism?*
}

\author{
M. S. Lourenço \\ University of Lisbon
}

\section{Introduction}

Good afternoon, ladies and gentlemen. Let me start by saying how I interpret this meeting, because that does determine what I am going to say next. I think about this meeting as a kind of technology fair, where products are for the first time shown and tested. The purpose of this meeting, as I see it, is to bring together professional philosophers doing analytic philosophy and ask them to show their products, so that hopefully we end up not only with a map of who is doing what but also with an increased pride in having given an old profession a distinctly new profile.

I would like to say why I think this is important and for that purpose I will revert to my technology metaphor. The practice of analytic philosophy requires such an intensive training and such a peculiar skill that each philosopher becomes by its practice a specialist, in Taylor's sense of the word. This is a reversal of a long-standing tradition of the philosopher as a generalist and, as we all know, anybody who wants to do analytic philosophy has to give up any claims to being a generalist. However, this is not to be seen as unfortunate in any way, because it is simply a by-product, on one hand, of the size of the literature in each of the traditional philosophical domains and, on the other, of the modern diversity of methods used in each of them. But we all eventually reach a point where we have to recognize that there is something unprofessional about mastering everything.

${ }^{*}$ This text was presented by M.S. Lourenço as the inaugural lecture of the First Meeting of the Portuguese Society for Analytic Philosophy, ENFA1, in Coimbra 2002. It was decided to include this lecture in the present volume even though the author did not have the opportunity to revise it for publication, and that explains some imprecisions in the text.

Disputatio, Vol. III, No. 27, November 2009 
On the other hand, I hope to be able to use the tools of analysis to rebalance the alleged disadvantage of a relative loss of scope. This analysis will show that it is, at least in principle, always possible to reach an overview, because philosophical theories split up in what I will call local theories, i.e., domain variant and global theories, i.e., domain invariant. A conspicuous instance of this dual structure is the use of global theories, like logic, epistemology and ontology, in individual domains, so that it becomes natural to define a theory in Ethics as ethical realism or ethical idealism and a faulty reasoning in Aesthetics is called the intentional fallacy.

And so our work has to proceed towards a reconciliation of the specialist's intense focus with the attainment of a balanced overview.

\section{Open Questions and Strategic Options}

In that sense, I propose, as my contribution to this Meeting, to show the line of goods I am interested in. But in my lecture I shall not be really engaged in problem solving, because I will rather attempt to answer the following heuristic question: what makes it exciting for a philosopher to engage in structuralist philosophy?

As I share Paul Benacerraff's view that philosophy of mathematics is philosophy in its most abstract state, I will try to consider general reasons that a philosopher might be inclined to use and to accept as grounds for an engagement in structuralism.

I would like to identify two sets of reasons a philosopher might accept as grounds for such an engagement:

(i) The intrinsic interest of the open questions and

(ii) The obvious thrill in defining parametric or strategic options.

I start with the first set. I provide a short list of the best-known open questions so that I may say something as to why I find them interesting.

Question \# 1: The varieties of structuralism. Not only does Structuralism divide along the lines between categorical and modal logic, but there are also several structuralist positions on themes like truth, reference and ontology. What are we to make of this diversity? The structuralist answer to this question is that this diversity is no more damaging to an understanding of the structuralist position than 
the diversity of definitions of usual objects, like natural numbers, functions and sets, is to our understanding of these objects.

Question \# 2: The transformations of the Identity Problem. This problem had an initial treatment by Benacerraff in 'What Numbers Could Not Be,' which articulates the classical structuralist position, and it then migrated to the theory of patterns in the form of the disputed claim that patterns are not in the field of the identity relation. Should there be a definition of identity for patterns? If yes, then in what form?

Question \# 3: The relation between the concepts of categorical set and pattern. Contrary to what was usual in the history of science, here the mathematical formulation preceded the philosophical. This fact is not only a counterexample disproving old time Positivism (Comte's law) but it also forces an answer to the McLarty question as to whether structuralism is fulfilled or obviated by the mathematical theory, in the sense that there is no need for a philosophical theory of abstract structures.

Question \# 4: In what sense is the concept of a category a foundational concept? An answer to this question depends on what one is supposed to recognize as a foundation and in this direction there is already J.P. Marquis's perceptive analysis of the relation ' $S$ is a foundation for $T$,' where $S$ and $T$ range inter alia over mathematical theories. Since he is not satisfied with his own solution, we have good reason to look for a way to complement it.

I call a strategic option also a parametric option because I intend to refer with this term to a goal or a value that remains unchanged or unquestioned throughout the development of a theory. Example: formal evidence will be chosen as a goal, because it is assumed to be more reliable than intuitive or informal evidence.

In this sense, what are then the most pressing strategic options in Structuralism?

Strategic Option \#1: Abstractionism. A commitment to abstractionism entails a redefinition of the doctrine and the rejection of Dummett's formulation of abstractionist concept formation.

Here is Dummett's description of abstractionist concept formation. His main thesis is that if abstractionism were to be coherent 
there would be a unique formulation of abstractionist concept formation. But in fact for him there are three different abstractionist theories and therefore abstractionism is incoherent. Dummett orders the theories as follows:

Theory \#1: It is possible to attain an as yet new concept by contemplating a number of otherwise distinct objects.

Theory \#2: The attainment of the new concept (in \#1) is effected by abstracting from the properties differentiating the objects in question. This is achieved by diverting attention from those properties.

Theory \#3: The operation of abstraction (in \#2) can also generate abstract mental constructions, that is, abstract objects or structural objects. These lack all those properties abstracted from and have no others in their place.

We cannot accept that this formulation does any kind of justice to the abstractionist position, let alone that it shows that there are three different abstractionist theories.

If one analyses Theory \#1 one soon finds out that it actually has no content, since there is no satisfactory way to define the concept of 'contemplation of otherwise distinct objects.' Turning to thesis \#2 here the stumbling block is the definition of the psychological (at least binary) function 'diverting attention from $X$ to $Y$.' But even assuming the existence of a satisfactory account of 'diverting attention from $X$ to $Y$,' in which $X$ and $Y$ would have to range over properties, it still remains unexplained how the target property $Y$ is an abstract object or a structure, as it is guaranteed by theory \#3.

The original definition of abstraction by Dedekind is still unrivalled. It simply says that abstraction is the convention according to which it is always a function that determines which properties an object is going to have, so that we can only grasp the object via our understanding of the function.

Strategic option \#2. A commitment to Fregean abstract dualism, whereby we will accept the existence of abstract entities that are not themselves objects. The now classical examples of such entities are Fregean functions and Fregean concepts, as opposed to Fregean courses of values. In this framework, categories and patterns will belong with Fregean functions and concepts, since they are also unsaturated or incomplete and thus are also predicative, like concepts. 
Strategic option \#3: The Problem of existence. This concept is interpreted as meaning reliable knowledge about the entities being asserted to exist. In the theory of patterns, knowledge is to be recognized as reliable when it meets the requirements of the method of the wide reflective equilibrium. With this method, we arbitrate between competing claims to reliability based on different kinds of evidence, e.g., formal versus informal. As was already suggested, truth is defined in terms of the coherence theory.

\section{Varieties of Structuralism}

I turn now to my block of open questions and would like to begin by saying a few words about the varieties of structuralism. As it would be desirable to have a minimal definition of structuralism, applicable to all its varieties, I propose to define it informally as a doctrine according to which the object of mathematical thought and the content of a mathematical theory is the presentation of a structure.

We can avoid the obvious circularity of this definition by giving the word 'structure' its more usual meaning, as a domain of objects together with relations and functions defined in it. An example of such a structure is a group, defined as

(1) A non empty domain $G$, together with a binary function on $G$ denoted by $\circ$ and

(2) such that $\circ$ is associative and

(3) there exists a unique element $i$ which is an identity for $\circ$ satisfying the equalities, for all $x$ in $G$,

$$
\begin{aligned}
& x \circ i={ }_{x} \\
& i \circ x_{x}
\end{aligned}
$$

and

(4) For every element $x$ of $G$ there is a unique inverse $y$ with respect to $\circ$, satisfying the equalities

$$
\begin{aligned}
& x \circ y=i \\
& y \circ x=i
\end{aligned}
$$


If we now analyse this definition we soon meet our familiar objects set (this is the domain), relation and function. This provides a settheoretical concept of structure and makes it possible to talk about structures as mathematical objects themselves. The structure is then a $n$-tuple, in my example the ordered pair $\langle G, \circ\rangle$, in which case the thesis that the object of mathematics is the presentation of structures includes the conception of set theory as the canonical language of mathematics.

Charles Parsons believed that a definition of structuralism was threatened by the following dilemma: if we posit structures or patterns as prime objects, we are unfaithful to the structuralist main thesis according to which prime objects are only positions in patterns; but if we accept the set-theoretical framework we have to provide an account of sets that is structuralistically meaningful. In spite of the fact that this dilemma can be met in more than one way, I want to leave this topic by remarking that it cannot be surprising that a definition of structuralism runs the risk of being circular. This is due to the fact that the concept of structuralism stands or falls with the more general concept of function, and for this one, as Frege has shown, there is no noncircular definition.

By now the best known taxonomy of the structuralist family is due to Charles Parsons in his 1990 paper 'The Structuralist View of Mathematical Objects.' There we find a division into eliminative and noneliminative, which he also calls the metalinguistic form of structuralism.

Eliminative structuralism, associated with the names of Paul Benacerraff, Michael Resnik and Stewart Shapiro, holds that statements about natural numbers are really about any simply infinite sequence or progression. In general, statements about any kind of mathematical objects are general statements about structures of a certain kind and provide thereby the elimination of reference to those mathematical objects. Eliminative structuralism also includes if-thenism or Logicism, first associated with the name of Hilary Putnam, and includes also Modalism, the program of eliminating mathematical objects in favour of modalities.

Non-eliminative structuralism takes the language of mathematics at face value and does not require that the objects referred to be any more objectively determined than the language itself already specifies. Structure is a metalinguistic notion, like a model in which the domain is given by a predicate, and the relations and functions by more predicates and functional terms. In the literature, there is another well-known taxonomy due to Michael Dummett in his Frege: Philosophy of Mathemat- 
ics. There he divides structuralism, as a philosophical theory about the reference of mathematical terms, into weak and strong.

In weak structuralism, associated with Frege, the definition of a mathematical object includes applications and the structuralist tone is obtained from the fact that anything of suitable structure will be considered. His example is the definition of the real numbers. For Frege, the real numbers apply to any set of relations, producing what he calls a magnitude domain. The conditions for the domain are laid down but the real numbers, i.e., the objects being defined, are defined as extensions covering all such domains.

Dummett divides strong structuralism into hard headed, to be associated with the name of Paul Benacerraff, and the mystical, to be associated with the name of Michael Resnik. Whereas hard-headed structuralists allow structures that also have non-structural properties, mystics only allow structures with structural properties.

I sketch now a short chronology of more than one hundred years of structuralist literature. Structuralism begins at the end of the XIX century (1888) with Dedekind, in particular his essay 'Was sind und was sollen die Zahlen?' I locate the period of early structuralism in the middle of the $20^{\text {th }}$ century, with two of the survivors of the Hilbert program, Paul Bernays in 1950, and Wilhelm Ackermann in 1960. Classical Structuralism begins with Paul Benacerraff in 1965 with his essay 'What Numbers Could Not Be.' Also in 1965, Charles Parsons published his first structuralist work and by the end of the sixties, 1969, Quine's ontological relativity shows a striking kinship with the structuralist approach to reference. Michael Resnik's theory of patterns, first and second version, and Stewart Shapiro's 'Mathematics and Reality' turn up in the 80's and in 1990 Charles Parsons proposed his non-eliminative metalinguistic version of structuralism.

In spite of the fact that classical structuralism began roughly at the time that category theory established itself, the structuralist meaning of this new and related theory was only first hinted at by John Bell in his 1980 lecture 'Category Theory and the Foundations of Mathematics' and then fully characterized in 1993 by Colin Mc Larty in a rival paper to 'What Numbers Could Not Be' with the title 'Numbers Can Be Just What They Have To.' A very perceptive analysis of the dispute as to which of the two concepts, category or set, is more appropriate for foundations, is to be found in J.P. Marquis's 1995 essay 'Category Theory and the Foundations of Mathematics: Philosophical Excavations.' 


\section{The Identity Problem}

Identity as a problem makes its first appearance in classical structuralism in connexion with Frege's difficulties with identities of the form

$$
n=\text { Julius Caesar. }
$$

To report on the eliminative position on identity I shall use expressions with the general form

$$
n=s
$$

in which $n$ is a cardinal number and $s$ a set.

Example: $5=\{\{\{\{\{0\}\}\}\}\}$.

Relative to expressions of this form Frege's dual problem was

(i) To compute always a truth-value for such identities and

(ii) To determine the meaning for the insertion in $s$ of a name or also of a definite description.

We can split such expressions in types, with basically 3 items:

Type 1: An arithmetical term is inserted in the right hand side of the identity.

Ex.: $5=2 \cdot 2+1$

Type 2: A term is inserted.

Ex.: $5=$ the number of continents

Type 3: A proper name is inserted.

Ex.: 5 = Julius Caesar.

Recall that for Frege there is a universe $U$ that contains the denotations of all names and definite descriptions. Thus, it makes sense for Frege to ask of any two names $n_{1}$ and $n_{2}$ whether they are the names of the same object. Frege complains that from the system of definitions in the Grundlagen he cannot decide his problem (ii) for expres- 
sions of type 3, so that he could not fix the meaning of identities of the form

$$
5=\text { Julius Caesar. }
$$

The Archimedean point of eliminativism, as far as identity is concerned, consists in denying that one always has to determine meaning in any expression with an occurrence of the binary symbol ' $=$ '. Instead, it must be possible to consider expressions of type 3 as asemantic or meaningless, in the same sense that one considers meaningful to say that ' 5 ' is the solution of the equation

$$
x \cdot 4=20
$$

and meaningless to say that 'Julius Caesar' is the solution of the same equation.

In eliminativism, an identity is only meaningful if one has a theory in whose language one can formulate and interpret individuation clauses. In general,

$$
a=b
$$

is meaningful if there is a class of objects $K$, which contains both $a$ and $b$ and such that the set of definitions which allow the recognition of a and $b$ as being the same $K$ also allows the computation of the truthvalue for

$$
a=b .
$$

If $a$ and $b$ are names of horses, we can decide whether they are the same horse. Individuation parameters are here names of parents, date of birth, place of birth etc. And if $a$ and $b$ are both names of classical gods we can also decide whether they are the same god. But the individuation parameters of a classical god cannot be the same as those of a horse, since what makes an object be a particular horse cannot make it be a particular classical god. In the end, the meaning of the question as to whether two objects $a$ and $b$ are indeed the same object is derived from the implicit assumption that $a$ and $b$ both belong to a certain fixed $K$. And so what is constitutive of the identity of an object is $K$ dependent, or rather dependent on the theory in which $K$ is used. 
It remains to consider the possibility of, instead of determining a meaning for formulas of type 3, to rather compute their truth-value in the sense of Frege's problem (i) above. Since we cannot have identities across theories, formulas of type 3 can only be false.

But as it is not easier to see that they are false rather than meaningless, in eliminativism one argues that they are either false or meaningless.

I would like to sketch now the identity problem in the theory of patterns and for that purpose I begin by explaining the basic vocabulary.

A pattern $P$ is a composite entity defined by a relation $R$ over the objects of $P$, called the points of the pattern. Patterns are also called structures and points are also called positions. The notation for a pattern is the notation for a structure, as already used. Thus

$$
\langle N, s\rangle
$$

denotes a pattern, whose points are the natural numbers and whose relation is the successor function. A pattern is not a set and differs from a set in that the pattern is not defined via its points, as a set is defined via its elements, but only by the relation $R$. The objects of $P$, the points or positions, have no identifying properties beyond the ones imposed by the relation $R$ and so no extra structural property defines identity.

The paradigm instance of a point or a position is actually the point of the plane. If you consider the unit circle with centre $c$ and diameter $a b$, the three points $a, b, c$ considered in isolation have no individuating properties, but as elements of the circle they can be distinguished. In a pattern, the only individuating properties of the objects are created by the relation, as it is the case with points in geometry, like the point $c$ that is the centre of our unit circle above.

Patterns can be in two kinds of relations:

(i) The first kind is the relation from pattern to instance, called instantiation, and

(ii) The second kind is the relation from pattern to pattern.

Of this second kind the following, ordered from weak to strong, are relevant:

Occurrence,

Mutual occurrence, 
Inclusion,

Equivalence,

Congruence.

From these the strongest is congruence, which is the relation that obtains between two patterns $P_{1}$ and $P_{2}$ which have isomorphic models or instances. I call two models isomorphic if there is a structure preserving function with a structure preserving inverse.

We now turn to Occurrence, which is the relation that obtains between two patterns $P_{1}$ and $P_{2}$ when $P_{1}$ is isomorphic to a pattern definable in $P_{2}$.

As an example, we can take $P_{1}$ to be $\langle N, s\rangle$ and $P_{2}$ to be $\langle Q+, x\rangle$.

A pattern $P_{1}$ is a subpattern of a pattern $P_{2}$ if every point in $P_{1}$ is in $P_{2}$ and $P_{1}$ occurs in $P_{2}$.

Occurrence is reflexive and transitive and thus is a quasi order.

If you now consider the following two patterns,

$$
\begin{aligned}
& P_{1}=\langle N, s\rangle \\
& P_{2}=\langle N,<\rangle
\end{aligned}
$$

it is obvious that every point in $P_{1}$ is in $P_{2}$ and that we can define 'successor' in $P_{2}$, by saying that

$n$ is the successor of $m$ if and only if $m<n$ and there is no $t$ such that $m<t<n$.

Since identity is itself an equivalence relation it is natural to search in the list for equivalence relations that could be seen as a proxy identity for patterns. The equivalence relations are: mutual occurrence, equivalence and congruence. As this last one is the strongest of the three, it suffices to show that identity is not equivalent to congruence.

It is enough to show that congruence cannot be a sufficient condition for identity between patterns. As a reductio argument, let us assume that it is. Then as the $N$-progression and the $2 \times N$ progression are congruent, by hypothesis they are identical. Therefore, their points are identical and we then have equalities like $1=2$. So congruence cannot be a sufficient condition for identity.

We could try to promote the use of the relation Occurrence as a definition of identity by interpreting occurrence as interpretability of theories, using definitions. 
We say that a theory $T_{1}$ is interpretable in a theory $T_{2}$ just in case there is a sequence of definitions of the primitive symbols of $T_{1}$ in $T_{2}$, which we denote by

$$
\left\{D T_{1} T_{2}\right\}
$$

which added to $T_{2}$ produces the theorems of $T_{1}$ as theorems of the new theory

$$
T_{2}+\left\{D T_{1} T_{2}\right\}
$$

We work this out with an example.

We assume two second-order theories $N^{\prime}$ and $N+$ with the following primitive symbols:

(i) $\quad N^{\prime}$ has 0 and monadic '

and

(ii) $\mathrm{N}+$ has 1 and dyadic + .

The axioms of $N^{\prime}$ are

(i) 0 is not a successor;

(ii) Successor is a 1-1 function;

(iii) Second-order induction.

The axioms of $\mathrm{N}+$ are the same, replacing the monadic successor function by ' +1 ' and defining 0 as the number whose successor is 1 . An so we have both:

(i) $N^{\prime}$ interpretable in $N+$.

The definition needed is $x^{\prime}=x+1$.

(ii) $N+$ is interpretable in $N^{\prime}$.

Two definitions will do, one in which 1 is defined as the successor of 0 and the other an inductive definition of $a+b=c$.

This will show that every model of $N^{\prime}$ has an occurrence of $N+$ inside and that this relation is symmetrical. So both theories are recip- 
rocally interpretable. However, they cannot be regarded as being the same, because $N^{\prime}$ is complete and categorical and $N+$ is neither complete nor categorical.

I sketch now the outline of the problem concerning the restriction of identity to a relation within a pattern. To carry out the ongoing analogy with geometry, we start by considering the possibility of developing geometry not as a theory of space but rather as a cluster of theories about configurations in some space.

In this framework, the points are now the positions within the configurations but not in the including space. We then define identity relative to objects of the configuration. In the ideal case there will be a separate theory for each configuration and in this case each theory will have an absolute identity predicate, ranging over all the elements of its universe.

If we apply now this analogy to arithmetic, we can certainly say that it deals with a certain configuration, and the identity of numbers can be taken as absolute in the sense that for any $x$ and $y$ in $N$ either $x=y$ or $x \neq y$.

There will be a slight ambiguity in the notation of relativized identity symbols. We can introduce the name of the theory or of the configuration as a subscript in $=$. If we now interpret the elements of the $N$ progression $0,1,2,3, \ldots$ to be positions, we can make sense of a statement $2 \neq_{n} 1$ as meaning that they are different positions of the $N$ progression; and about the progression $0,2,4,6, \ldots$ we may want to express that 2 has in the e-progression the position that 1 has in the $n$ progression by writing $2={ }_{e} 1$. But then within the same pattern one will have formulas like $4={ }_{e} 2$, to express that 4 has in the e-progression the position that 2 has in the $n$-progression, but we also have $4 \neq_{e} 2$ to express that 4 and 2 are different points of the same e-progression.

It would appear to have been more natural to try to define identity between patterns by a formula like

$P_{1}$ is the same as $P_{2}$ if and only if

for every $x, x$ is a position of $P_{1}$ if and only if $x$ is a position of $P_{2}$ and the relations of $P_{1}$ are extensionally identical to the relations of $P_{2}$.

Such a formulation would however have the disadvantage of a commitment to admitting positions of different patterns into a single universe of discourse. 
Therefore, patterns and positions in different patterns are not in the field of the identity relation. In conclusion, what are then the options for cross pattern relations? There are at least three possible options.

The first is to accept a many-sorted framework in which each pattern would have its own separate universe of discourse. The second would be exploring again the analogy with geometry and positing a space of positions or points, from which patterns could be constructed. Identity in this framework would then apply to all points of this space, but then of course the theorems about this space would not deal with individual points.

In principle, there is a third and last possibility, which is with the framework of set theory. The points of a pattern would be conceived as sets and the theory of patterns would be reduced to set theory. The obvious disadvantage of this proposal is to force the interpretation of patterns as sets, which is something that they are not.

What does the rejection of congruence as the identity relation for patterns amount to? It amounts to the following. If two patterns are congruent then they are isomorphic, and isomorphic objects share all the same properties. But by Leibniz's conception of identity we cannot have distinct objects with no difference between them. So if those two patterns are different, but yet indiscernible, they violate Leibniz's identity law. Shall we then interpret this as meaning that Leibniz's law of identity is to be rejected or as meaning that the congruence relation has to count as identity?

It turns out that Leibniz's identity law is not the only conception of identity available. Less known is the so called Behmann's identity, also followed by Ramsey and the early Wittgenstein, according to which two objects can be different and yet satisfy exactly the same properties. Behmann's identity would then make justice to the theory of patterns by allowing two patterns to be different and yet be isomorphic or congruent.

There is a simple logical relation between Leibniz's identity and Behmann's identity and that is that a formula expressing Behmann's identity is the dual of a formula expressing Leibniz's identity. In this case one could say that pattern congruence is the dual form of identity. I close this topic by remarking that even this solution leaves an after taste of an ad hoc expedient because both conceptions of identity are supposed to handle identity between objects. But since patterns are not objects, we should not expect any known form of identity to hold for them. 


\section{From Patterns to Categories}

In his paper on Cantor's Continuum Problem, Gödel underlines the shift in conceptual scheme that occurred with the introduction of Ellmann and McLane's theory of categories in relation to the iterative conception of set. I would like to say something about this shift and sketch the outlook of the new conceptual scheme before comparing the structuralist with the categorical point of view.

To explain the notation: if $g$ is a function from a set $A$ to a set $B$ and $f$ a function from the set $B$ to a set $C$, then by

$$
f \circ g
$$

I mean the function $h$ from $A$ to $C$ such that

$$
H(x)=f(g(x)) .
$$

We call $f \circ g$ the composition of both functions.

This notation is equivalent to $f \circ g(x)$. If we define an isomorphism between two sets $A$ and $B$ to be a function $f$ from $A$ to $B$ with an inverse $f^{1}$ from $B$ to $A$, then the composite

$$
f^{1} \circ f
$$

is the identity function on $A$, and

$$
f \circ f^{1}
$$

is the identity function on $B$.

As far as terminology is concerned, a relation in a category will be called usually a morphism, but the notion matches in many examples the profile of a function in the usual sense. The simplest informal description of a category $C$ is as a totality $O$, which we posit to contain the objects of $C$. We characterize the totality $O$ by the following existence statements:

(i) For every pair $A, B$ of elements of $O$, there is a totality $M(A, B)$, called the morphisms from $A$ to $B$ in $C$;

(ii) For every triple of elements $A, B, C$ in $O$ there is an operation from pairs of morphisms in 


$$
M(A, B) \times M(B, C)
$$

to morphisms in $M(A, C)$ called the composition of morphisms in $C$. In the notation above, if $f$ is a morphism from $A$ to $B$ and $g$ a morphism from $B$ to $C$ then

$$
g \circ f
$$

is the morphism from $A$ to $C$, their composition;

(iii) For every element $A$ in $O$ there is in $M(A, A)$ a morphism Id $A$, called the identity on $A$.

From morphisms two things are in turn required:

(1) The associativity of their composition, and

(2) left and right identities.

Thus if $f$ is a morphism from $A$ to $B$ and $g$ a morphism from $B$ to $C$ and $h$ a morphism from $C$ to $D$, then we want the equality

$$
h \circ(g \circ f)=(h \circ g) \circ f
$$

to be true.

The left and right identities are captured in the two formulas

$$
\left(\operatorname{Id}_{\mathrm{B}} \circ f\right)=f
$$

and

$$
\left(f \circ \mathrm{Id}_{\mathrm{A}}\right)=f \text {, }
$$

when $f$ is a morphism from $A$ to $B$. The best-known examples of mathematical structures are examples of categories. The philosophically interesting feature of this concept is that what distinguishes a category is the category's own morphisms, not the category's own elements. In a category's instance these elements are structured sets with structure preserving maps. However, a category is anything that fulfils the definition above. For my purpose, I use abstract sets as an example of a category. Whereas concrete sets collect concrete individuals together, an abstract set is an idealization of a concrete set 
with the significant difference that it is described by its functions rather than by its concrete elements. So if $A$ and $B$ are abstract sets and $f$ a function from $A$ to $B$ then any function $g$ from $B$ to $C$ has a composite with $f, g \circ f$ from $A$ to $C$. Notice that we do not define composition by an action on the elements of $A$. We can now have axioms to stipulate the associativity of composition and for the existence of the identity functions. In particular, every set $A$ has a function $\mathrm{Id}_{\mathrm{A}}$ from $A$ to $A$ such that the following identities are true:

$$
\begin{aligned}
& f \circ \operatorname{Id}_{\mathrm{A}}=f \text { and } \\
& \mathrm{Id}_{\mathrm{A}} \circ h=h,
\end{aligned}
$$

when $f$ is a function from $A$ to $B$ and $h$ is a function from $C$ to $A$. Thus abstract sets and functions are an instance of a category.

At this point, it is useful to introduce an axiom guaranteeing the existence of the abstract singleton. This axiom asserts the existence of a set, denoted by 1 , with the following property: no set can have more than one function with range 1 . It is then possible to define abstract element as being actually also a function. Thus an element $x$ of an abstract set $A$ is any function $x$ from 1 to $\mathrm{A}$, and as such elements have also only structural properties.

This shift from object (or element) to function is the essence of the new categorical outlook, first described by Lawvere in 1964. In categorical set theory sets and functions have only structural properties. And as I said above we have two ways to interpret this result: either so that it would seem that the structuralist program is thereby fulfilled or so that it would seem that the structuralist program is thereby made redundant.

I take the easiest disjunct first, the one about redundancy. To define a theory as redundant relative to another it has to be assumed that they are both theories of the same kind pursuing the same goals. Now, this is not the case in the present debate, since structuralism does not offer a mathematical theory that can compete with categorical set theory. In this sense, structuralism is not redundant and it has to be justified on its own merits, as any standard philosophical theory.

It remains to settle the question of whether categorical set theory fulfils the structuralist program. Here the whole question rotates around the interpretation of the concept of fulfilling a program. There was obviously no structuralist program around when categories and abstract sets appeared and produced the shift that Gödel mentions 
in his article. So, in this interpretation there is no fulfilment of the structuralist program. But there is another interpretation possible, namely as viewing the philosophical theory as an expedient rhetorical device that captures the spirit and the achievements of categories and categorical set theory.

M. S. Lourenço University of Lisbon 